\title{
Direct observation of dramatically enhanced hole formation in a perovskite-solar-cell material spiro-OMeTAD by Li-TFSI doping
}

Miki Namatame, Masaki Yabusaki, Takahiro Watanabe, Yuhei Ogomi, Shuzi Hayase, and Kazuhiro Marumoto

Citation: Appl. Phys. Lett. 110, 123904 (2017); doi: 10.1063/1.4977789

View online: http://dx.doi.org/10.1063/1.4977789

View Table of Contents: http://aip.scitation.org/toc/apl/110/12

Published by the American Institute of Physics

\section{Articles you may be interested in}

Mechanical signatures of degradation of the photovoltaic perovskite $\mathrm{CH} 3 \mathrm{NH} 3 \mathrm{Pbl} 3$ upon water vapor exposure Appl. Phys. Lett. 110, 121903121903 (2017); 10.1063/1.4978687

Ferroelectric, pyroelectric, and piezoelectric properties of a photovoltaic perovskite oxide Appl. Phys. Lett. 110, 063903063903 (2017); 10.1063/1.4974735

Current-induced surface roughness reduction in conducting thin films Appl. Phys. Lett. 110, 103103103103 (2017); 10.1063/1.4977024

Enhanced energy storage density by inducing defect dipoles in lead free relaxor ferroelectric BaTiO3-based ceramics

Appl. Phys. Lett. 110, 132902132902 (2017); 10.1063/1.4979467

Measuring hole spin states of single quantum dot in germanium hut wire

Appl. Phys. Lett. 110, 133105133105 (2017); 10.1063/1.4979521

Modulation of electronic and optical properties in mixed halide perovskites $\mathrm{CsPbCl} 3 \times \mathrm{Br} 3(1-\mathrm{x})$ and CsPbBr3xI3(1-x)

Appl. Phys. Lett. 110, 113901113901 (2017); 10.1063/1.4978598

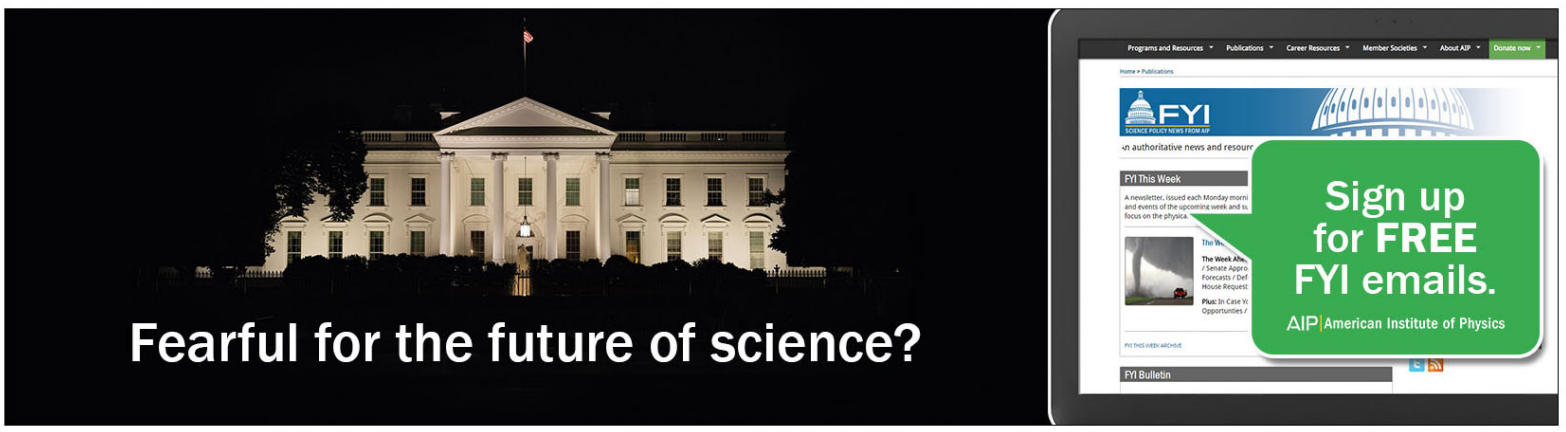




\title{
Direct observation of dramatically enhanced hole formation in a perovskite-solar-cell material spiro-OMeTAD by Li-TFSI doping
}

\author{
Miki Namatame, ${ }^{1}$ Masaki Yabusaki, ${ }^{1}$ Takahiro Watanabe, ${ }^{1}$ Yuhei Ogomi, ${ }^{2}$ Shuzi Hayase, ${ }^{2}$ \\ and Kazuhiro Marumoto ${ }^{1,3, a)}$ \\ ${ }^{1}$ Division of Materials Science, University of Tsukuba, Tsukuba, Ibaraki 305-8573, Japan \\ ${ }^{2}$ Graduate School of Life Science and Systems Engineering, Kyushu Institute of Technology, \\ Fukuoka 808-0196, Japan \\ ${ }^{3}$ Tsukuba Research Center for Interdisciplinary Materials Science (TIMS), University of Tsukuba, Tsukuba, \\ Ibaraki 305-8571, Japan
}

(Received 27 December 2016; accepted 13 February 2017; published online 21 March 2017)

\begin{abstract}
Electron spin resonance (ESR) spectroscopy of 2,2',7,7'-tetrakis-( $N, N$-di-p-methoxyphenylamine) $9,9^{\prime}$ spirobifluorene (spiro-OMeTAD) thin films and perovskite $\left(\mathrm{CH}_{3} \mathrm{NH}_{3} \mathrm{PbI}_{3}\right)$ /spiro-OMeTAD layered films are reported. Clear ESR signals $(g=2.0030)$ were observed by adding a dopant lithium bis(trifluoromethanesulfonyl)imide (Li-TFSI) to the spiro-OMeTAD thin films, which directly showed the spin (hole) formation in spiro-OMeTAD by the Li-TFSI doping. The number of spins in the spiroOMeTAD thin film has increased by more than two orders of magnitude by the Li-TFSI doping under dark conditions, which demonstrates from a microscopic viewpoint that Li-TFSI has high doping effects for the spiro-OMeTAD thin films. Under simulated solar irradiation, the spin density in the spiro-OMeTAD thin films and the perovskite/spiro-OMeTAD layered films largely increased by the Li-TFST doping due to the formation of long-lived holes in spiro-OMeTAD. The transient responses of the number of photogenerated spins, $N_{\text {spin }}$, of the layered films upon the light irradiation showed the increase and the decrease in the $N_{\text {spin }}$ due to the hole transfer and recombination at the perovskite/spiro-OMeTAD interface. The states of long-lived holes in the spiro-OMeTAD layers were analyzed using the simulation of the ESR spectra, which reveals the mobile photogenerated holes with a lifetime $>10 \mu$ s. Published by AIP Publishing.
\end{abstract}

[http://dx.doi.org/10.1063/1.4977789]

Perovskite solar cells are capable of being produced by solution methods and have attracted much attention as a lowcost solar cell. ${ }^{1-4}$ Recently, the power conversion efficiencies of over $20 \%$ have been reported, and the study for further efficiency improvement has been actively conducted because of the expectation as a new solar cell to replace inorganic solar cells. ${ }^{5,6}$ The perovskite materials have been used as photovoltaic active layers in the devices, which have shown the light absorption with a wide wavelength region ${ }^{7,8}$ and the photogeneration of free electron and hole carriers without forming excitons. ${ }^{9}$ However, the hole-transporting materials in the devices have not yet been fully investigated, which is also one of the important studies of the perovskite solar cells. As a typical hole-transporting material for the perovskite solar cells, $2,2^{\prime}, 7,7^{\prime}$-tetrakis-( $N, N$-di- $p$-methoxyphenylamine $) 9,9^{\prime}$-spirobifluorene (spiro-OMeTAD) has been used. ${ }^{1-4}$ Lithium bis(trifluoromethanesulfonyl)imide (Li-TFSI) has been utilized as a dopant for spiro-OMeTAD; ${ }^{2}$ this doping technique has been employed for the dye-sensitized solar cells. ${ }^{10}$ The performance of the perovskite solar cells with spiro-OMeTAD has been reported to be greatly improved by the Li-TFSI doping because of the improvement of the conductivity in spiroOMeTAD. ${ }^{11,12}$ However, the improvement mechanism of the conductivity in spiro-OMeTAD by the Li-TFSI doping has not yet been fully studied from a microscopic viewpoint, and

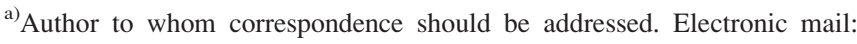
marumoto@ims.tsukuba.ac.jp
}

the details of the charge states in spiro-OMeTAD have not yet been completely clarified.

For a microscopic investigation of the charge states in organic devices and their constituent materials, electron spin resonance (ESR) spectroscopy is one of the most useful methods. ${ }^{13-18}$ The ESR spectroscopy can identify the molecules where charges with spins exist and can evaluate the absolute value of the number of spins. ${ }^{13-18}$ Thus, it has been considered that the ESR study of the perovskite solar cells and their constituent-material spiro-OMeTAD is important for obtaining the knowledge of the charge states and the improvement mechanism of the device performance. However, such detailed study has not yet been carried out using the ESR spectroscopy.

Here, we report the ESR study of the spiro-OMeTAD thin films and the perovskite/spiro-OMeTAD layered films to investigate the charge states in the constituent materials in the perovskite solar cells. The dramatic enhancement of the ESR signal of the spiro-OMeTAD thin films was observed by the Li-TFSI doping effects. Moreover, the existence of long-lived charge separation due to the perovskite/spiro-OMeTAD interface was confirmed from a microscopic viewpoint. From the Li-TFSI doping effects in spiro-OMeTAD, the improvement mechanism of the electrical conductivity is explained by the fillings of the deep trapping sites in spiro-OMeTAD, leading to the improvement of the device performance.

Nonmagnetic quartz/ $/ \mathrm{Al}_{2} \mathrm{O}_{3}$ substrates $(3 \mathrm{~mm} \times 20 \mathrm{~mm})$ were utilized to fabricate the samples of quartz/ $/ \mathrm{Al}_{2} \mathrm{O}_{3} /$ spiroOMeTAD $(\sim 100 \mathrm{~nm}$, with or without Li-TFSI) thin films 
and quartz/perovskite $(\sim 400 \mathrm{~nm}) /$ spiro-OMeTAD $(\sim 100 \mathrm{~nm}$, with or without Li-TFSI) layered films for the ESR measurements. In the following, these samples are abbreviated as the spiro-OMeTAD thin film and the perovskite/spiro-OMeTAD layered film, respectively, which were fabricated following the literatures. ${ }^{8,19}$ All samples were naturally oxidized under ambient air conditions outside the glove box after the fabrication. The details of the fabrication process are described in supplementary material. The fabricated samples were sealed in an ESR sample tube with helium gas at 100 Torr after evacuating the tube below $6 \times 10^{-4} \mathrm{~Pa}$. Only the spiroOMeTAD thin film without Li-TFSI was sealed in a nitrogen-filled glove box. The ESR measurements were carried out with a JEOL RESONANCE JES-FA200 X-band spectrometer under dark conditions or simulated solar irradiation using a Bunkoukeiki OTENTOSUN-150LX solar simulator at room temperature. The number of spins, the $g$ value, and the linewidth of the ESR signals were calibrated using a standard $\mathrm{Mn}^{2+}$ marker sample.

The ESR spectroscopy is useful to directly and quantitatively investigate the charge formation by doping from a microscopic viewpoint. First, the ESR measurements were carried out for the spiro-OMeTAD thin films to study the LiTFSI doping effects. Figure 1(a) shows the dependence of the ESR spectra of the spiro-OMeTAD thin film on the LiTFSI doping. The vertical axis is plotted using a unit of the peak-to-peak ESR intensity of the standard $\mathrm{Mn}^{2+}$ marker sample, $I_{\mathrm{Mn}}$. The ESR signal of the spiro-OMeTAD thin film without Li-TFSI was hardly observed because the number of spins was small, less than $2.8 \times 10^{11}$. Although the spiroOMeTAD thin film was naturally oxidized after the fabrication, the doping effect hardly occurred by the natural oxidation. In contrast, a clear ESR signal of the spiro-OMeTAD thin film was observed by the Li-TFSI doping. The obtained ESR parameters were as follows: the $g$ factor of $g=2.0030 \pm 0.0002$, the peak-to-peak ESR linewidth of $\Delta H_{\mathrm{pp}}=842 \pm 10 \mu \mathrm{T}$, and the number of spins of $5.2 \times 10^{13}$. The obtained $g$ factor is well consistent with that of the radical species of the spiro-OMeTAD powder samples doped with H-TFSI. ${ }^{20}$ Notably, the number of spins is increased by more than two orders of magnitude by the Li-TFSI doping. For the doping states of spiro-OMeTAD, the formation of cations of spiro-OMeTAD(TFSI) and dications of spiro$\mathrm{OMeTAD}(\mathrm{TFSI})_{2}$ has been discussed. ${ }^{12}$ If the cations are formed, the observed ESR signal is reasonably ascribed to the radical cations in the spiro-OMeTAD thin film. If the dications are formed, although the dications of conventional molecules are nonmagnetic, the observed ESR signal may indicate the formation of diradical dications with magnetism in the spiro-OMeTAD thin film, which is most likely due to the torsion of the spiro structure of a spiro-OMeTAD molecule. A zero-field splitting of the ESR signal due to triplet diradicals was not clearly observed at around $g \sim 2$, which may be attributed to the long distance between radical pairs due to the spiro structure mentioned above. Thus, we could not identify the doping states of spiro-OMeTAD whether cations or dications from the ESR signals. We here evaluate the doping concentration of spiro-OMeTAD by Li-TFSI when the radical cations of spiro-OMeTAD(TFSI) or the diradical dications of spiro-OMeTAD(TFSI $)_{2}$ are formed. From the
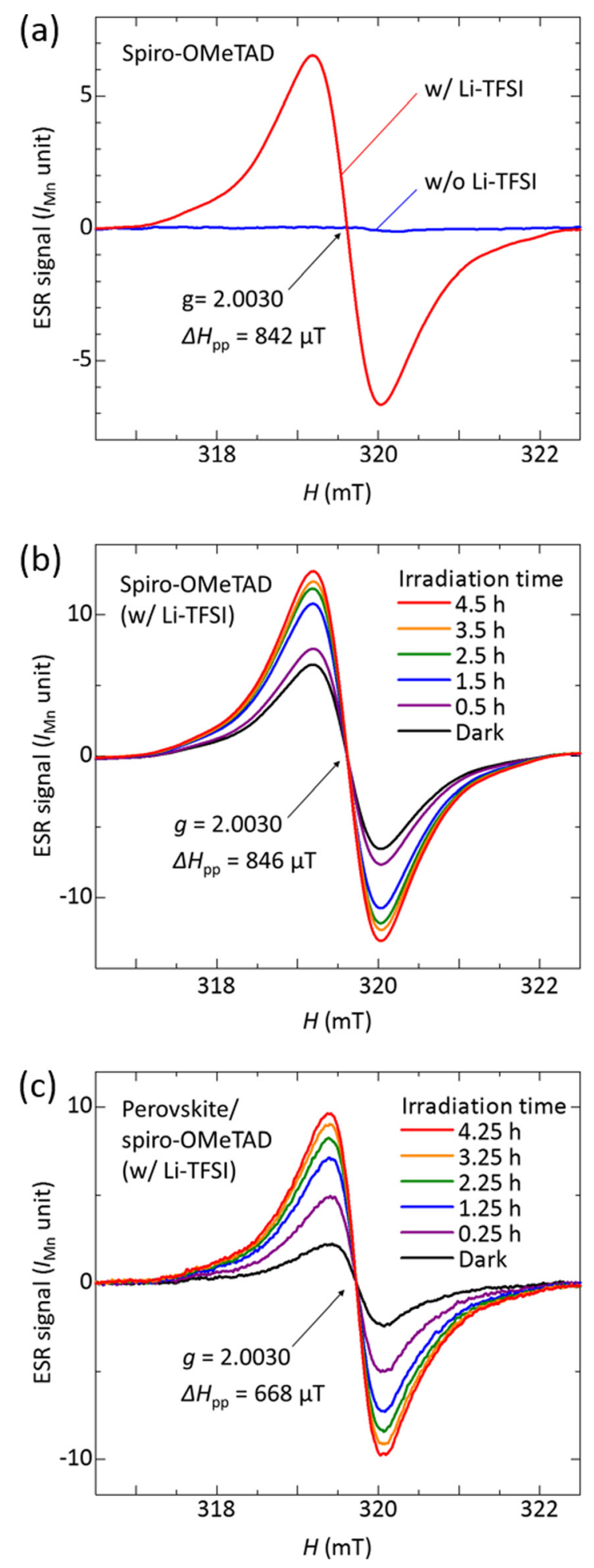

FIG. 1. (a) Dependence of the ESR spectrum of the spiro-OMeTAD thin film on the Li-TFSI doping. ESR measurements were carried out under dark conditions. (b) and (c) The ESR spectra of (b) the spiro-OMeTAD thin film with Li-TFSI and (c) the perovskite/spiro-OMeTAD (with Li-TFSI) layered film for various exposure times to simulated solar light (AM 1.5G) with a $100 \mathrm{~mW} \mathrm{~cm}^{-2}$ intensity at room temperature.

volume of the spiro-OMeTAD thin film of $6.0 \times 10^{-6} \mathrm{~cm}^{3}$, the doping concentration per a spiro-OMeTAD monomer is evaluated to be approximately $0.90 \%$ and $0.45 \%$ from the formation of radical cations and diradical dications, respectively, using the density of the spiro-OMeTAD thin film of $1.82 \mathrm{~g} \mathrm{~cm}^{-3} .^{21}$ The Li-TFSI doping effect may be explained 
by the formation of lithium oxide because the spiro-OMeTAD layers were naturally oxidized after the fabrication. ${ }^{11}$

To clarify the formation and the accumulation of the charges under light irradiation, the light-induced ESR measurements were carried out for the spiro-OMeTAD thin films. In our ESR measurements, a continuous-wave method with a modulation frequency of $100 \mathrm{kHz}$ was used for the external magnetic field $H .{ }^{16}$ Thus, the photogenerated charge carriers with a lifetime of $<10 \mu$ s cannot be observed using the present ESR method. ${ }^{16}$ The observed light-induced ESR signals are due to the photogenerated charge carriers with a lifetime of $>10 \mu \mathrm{s}$, namely, accumulated (or deeply trapped) photogenerated carriers. ${ }^{16}$ Figure 1(b) shows the dependence of the ESR spectra of the spiro-OMeTAD thin film with LiTFSI on the duration of simulated solar irradiation. The ESR signal gradually and monotonically increased with the increasing duration of simulated solar irradiation. The obtained ESR parameters were $g=2.0030 \pm 0.0002$ and $\Delta H_{\mathrm{pp}}=846 \pm 10 \mu \mathrm{T}$. Since the obtained ESR parameters under the light irradiation are coincided with those obtained under dark conditions shown in Fig. 1(a), the observed charges under the light irradiation are ascribed to the holes (radical cations or diradical dications) of spiro-OMeTAD. Since the band gap of spiro-OMeTAD is $2.94 \mathrm{eV},{ }^{22}$ the lightinduced ESR signals originate from the charge separation due to the light absorption in high energy region of simulated solar irradiation. In contrast, the ESR signal of the spiroOMeTAD thin film without Li-TFSI slightly increased by simulated solar irradiation, and the signal intensity remained as a small constant during the light irradiation. Therefore, we conclude that the Li-TFSI doping remarkably enhances the formation and the accumulation of long-lived charges due to the charge separation in the spiro-OMeTAD thin films under the light irradiation.

Since the hole transfer at the interfaces between perovskite and spiro-OMeTAD is a very interesting issue, the lightinduced ESR measurements were carried out for the perovskite/spiro-OMeTAD layered films. Figure 1(c) shows the dependence of the ESR spectra of the perovskite/spiroOMeTAD (with Li-TFSI) layered film on the duration of simulated solar irradiation. The increase in the ESR signal with the increasing duration of the light irradiation was observed. The ESR parameters were obtained as $g=2.0030 \pm 0.0002$ and $\Delta H_{\mathrm{pp}}=668 \pm 10 \mu \mathrm{T}$. The $g$ factor is consistent with that of the holes of spiro-OMeTAD mentioned above. The lightinduced ESR signal due to perovskite was not identified at room temperature, which may indicate the absence of longlived charges in perovskite and/or the no detection of the ESR signal due to the rapid spin relaxation of charges in perovskite at room temperature. ${ }^{9,16,23}$

To investigate the transient response of the ESR signals upon the light irradiation, the variation of the number of spins was evaluated. The number of photogenerated spins, $N_{\text {spin }}$, was evaluated by integrating the light-induced ESR spectrum twice and by comparing with the standard $\mathrm{Mn}^{2+}$ marker sample; the light-induced ESR spectrum was obtained by subtracting the ESR spectrum under dark conditions from that under simulated solar irradiation or after the light irradiation. Figure 2 shows the transient responses of the $N_{\text {spin }}$ of the spiro-OMeTAD thin films and the

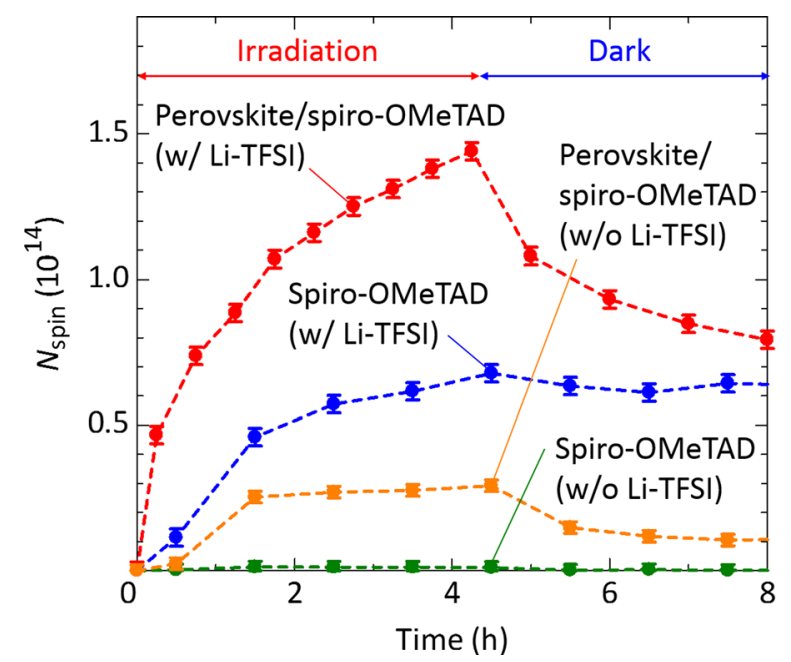

FIG. 2. Transient responses of the number of spins, $N_{\text {spin }}$, due to photogenerated holes in spiro-OMeTAD on the duration of simulated solar irradiation for the perovskite/spiro-OMeTAD (with Li-TFSI) layered film (red circles), the perovskite/spiro-OMeTAD (without Li-TFSI) layered film (orange circles), the spiro-OMeTAD thin film with Li-TFSI (blue circles), and the spiro-OMeTAD thin film without Li-TFSI (green circles).

perovskite/spiro-OMeTAD layered films. The $N_{\text {spin }}$ of the spiro-OMeTAD thin film without Li-TFSI slightly increased by the light irradiation. After the light irradiation, the $N_{\text {spin }}$ rapidly decreased to almost zero. In contrast, the $N_{\text {spin }}$ of the spiro-OMeTAD thin film with Li-TFSI monotonically increased by the light irradiation. This result shows that the Li-TFSI doping of the spiro-OMeTAD thin film enhances the formation of long-lived charges. Under dark conditions after the light irradiation, the $N_{\text {spin }}$ hardly decreased and showed an almost constant value. Notably, the $N_{\text {spin }}$ of the perovskite/spiro-OMeTAD (with Li-TFSI) layered film largely increased under the light irradiation compared with the case of the spiro-OMeTAD thin film with Li-TFSI. This increase is rationally ascribed to the hole transfer at the perovskite/spiro-OMeTAD interfaces, followed by the formation of long-lived charges with a long lifetime of $>10 \mu \mathrm{s}$. Also, the Li-TFSI doping is likely to enhance the hole transfer yield at the perovskite/ spiro-OMeTAD interfaces on the $10 \mu$ s time scale because the $N_{\text {spin }}$ of the perovskite/spiro-OMeTAD (with Li-TFSI) layered film showed a larger increase compared with the case without Li-TFSI under the light irradiation (see Fig. 2). This result is consistent with that obtained from the studies using the time-resolved microwave conductivity that have quantified the hole transfer yields from the perovskite to polymer or molecular hole-transport layers within a few microseconds. ${ }^{24,25}$ The $N_{\text {spin }}$ under dark conditions after turning off the light irradiation greatly decreased. This decrease is reasonably attributed to the recombination of the long-lived charges at the perovskite/spiro-OMeTAD interface. However, all photogenerated spins did not recombine; approximately half of the maximum $N_{\text {spin }}$ remained at $4 \mathrm{~h}$ later after turning off the light irradiation. Since the remained $N_{\text {spin }}$ value of the layered film is almost the same as that of the spiro-OMeTAD thin film with Li-TFSI at $4 \mathrm{~h}$ later after turning off the light irradiation, the origins of these spins are likely the same with each other. 
We now turn to a discussion of the origin of the remained spins in the spiro-OMeTAD thin film with Li-TFSI and the perovskite/spiro-OMeTAD (with Li-TFSI) layered film after the light irradiation. For the doping of the spiroOMeTAD thin films, spiro-OMeTAD(TFSI $)_{2}$ has been demonstrated to be effective for enhancing the hole conductivity in spiro-OMeTAD, where the formation of spiroOMeTAD(TFSI) in addition to spiro-OMeTAD(TFSI) ${ }_{2}$ has been discussed. ${ }^{12}$ Thus, the observed signals in this study may originate from the holes (cations or dications) of spiroOMeTAD. ${ }^{12}$ When the hole is formed in spiro-OMeTAD by the light irradiation, the anion and/or the dianion of spiroOMeTAD may be formed due to the photogeneration of hole and electron pairs. In this study, however, the ESR signal due to anions and/or dianions of spiro-OMeTAD were hardly observed. One possible reason for the hard formation of anions and/or dianions is due to the electron transfer from spiro-OMeTAD to complexes of $\mathrm{Li}^{+}$and $\mathrm{H}_{2} \mathrm{O}$, as explained below. ${ }^{22,26}$ The spiro-OMeTAD has a shallow lowest unoccupied molecular orbital (LUMO) level of $-2.28 \mathrm{eV} .{ }^{22}$ Also, it has been discussed that Li-TFSI has a strong interaction with water molecules resulting in the formation of the complexes of $\mathrm{Li}^{+}$and $\mathrm{H}_{2} \mathrm{O} .{ }^{26}$ Thus, the electrons formed by the light absorption in spiro-OMeTAD may be trapped in the $\mathrm{Li}^{+}-\mathrm{H}_{2} \mathrm{O}$ complexes, forming the ESR-silent species of $\mathrm{H}_{2}$ and $\mathrm{OH}^{-}$. Another possibility is the electron transfer from spiro-OMeTAD to adsorbed $\mathrm{O}_{2}$ in the films, forming oxygen radical anions $\mathrm{O}_{2}{ }^{-}$. The ESR signals due to adsorbed $\mathrm{O}_{2}$ were not observed because the resonance magnetic fields were out of the measured $H \cdot{ }^{27}$ Also, the ESR signals due to oxygen radical anions $\mathrm{O}_{2}{ }^{-}$cannot be observed at room temperature because of the broadening of the ESR linewidth resulting from the fast relaxation due to the orbital rotation of $\mathrm{O}_{2}{ }^{-28,29}$ In both cases, since the electrons trapped in the complexes and/or adsorbed $\mathrm{O}_{2}$ are hardly mobile and hardly contribute to the recombination, the holes in spiro-OMeTAD may remain without the recombination with the electrons.

It is interesting to analyze the motion of the long-lived holes formed in spiro-OMeTAD in detail. The analysis was performed with a fitting calculation using the Lorentzian and Gaussian functions. When a spin is mobile, the ESR lineshape is expressed by a Lorentzian function. When a spin is static, the ESR lineshape is expressed by a Gaussian function. For the spiro-OMeTAD thin films with Li-TFSI, the ESR lineshape was found to hardly change upon the light irradiation. This result indicates that the states of most of the long-lived holes hardly change upon the light irradiation. In contrast, for the perovskite/spiro-OMeTAD (with Li-TFSI) layered films, the ESR lineshape changed by the light irradiation. Figure S1 of supplementary material shows the fitting analysis for the light-induced ESR spectrum of the perovskite/spiro-OMeTAD (with Li-TFSI) layered film. Here, the blue open circles show the observed light-induced ESR spectrum, the orange and green dashed lines show the ESR spectra of the Lorentzian and Gaussian components, respectively, and the red solid line show the sum of these components. The $g$ factors for the Lorentzian and Gaussian components were obtained as $g=2.0032 \pm 0.0003$, respectively. Table I shows each evaluated ratio of the $N_{\text {spin }}$ of the Lorentzian and Gaussian components to the total $N_{\text {spin }}$. The light-induced
TABLE I. The ratio of the $N_{\text {spin }}$ of each component obtained from the fitting analysis for the ESR spectra of the perovskite/spiro-OMeTAD (with LiTFSI) layered film under dark conditions (ESR under dark), the lightinduced ESR (LESR) spectrum under the light irradiation for the duration between 4.0 and $4.5 \mathrm{~h}$ (LESR under irradiation), and the LESR spectrum under dark conditions after turning off the light irradiation for the duration between 3.5 and $4.0 \mathrm{~h}$ (LESR under dark after irradiation off).

\begin{tabular}{|c|c|c|}
\hline $\begin{array}{l}\text { ESR spectrum } \\
\text { for fitting analysis }\end{array}$ & $\begin{array}{l}\text { Ratio of } \\
N_{\text {spin }}(\%) \text { for Lorentzian } \\
\text { comp. }\end{array}$ & $\begin{array}{c}\text { Ratio of } N_{\text {spin }} \\
(\%) \text { for Gaussian comp. }\end{array}$ \\
\hline ESR under dark & 76 & 24 \\
\hline LESR under irradiation & 84 & 16 \\
\hline $\begin{array}{l}\text { LESR under dark } \\
\text { after irradiation off }\end{array}$ & 77 & 23 \\
\hline
\end{tabular}

ESR spectrum under the irradiation shows a larger ratio of the Lorentzian component compared with those of the other ESR spectra. Also, the ESR spectrum under dark conditions and the light-induced ESR spectra under dark conditions after turning off the irradiation show a similar ratio for the Lorentzian and Gaussian components. Therefore, these results demonstrate the increase in the ratio of the mobile long-lived holes with a long lifetime of $>10 \mu$ s in the spiro-OMeTAD films by the irradiation, which is ascribed to the hole transfer due to the perovskite/spiro-OMeTAD interfaces.

The long-lived holes in the spiro-OMeTAD films after the light irradiation may be important to understand the origins for hysteresis, degradation of the short circuit current after long-time irradiation, and durability, which are often observed in the perovskite solar cells using spiro-OMeTAD with Li-TFSI doping. To discuss the relation between longlived holes and such characteristics, we fabricated the cells by the similar methods used for this study. Figure S2 of supplementary material shows the current density $(J)$-voltage $(V)$ characteristics of a fabricated cell. We observed a hysteresis behavior and a lower performance under a forward sweep compared with that under a backward sweep, which may be ascribed to the motion of the long-lived holes in the spiro-OMeTAD film. Figure S3 of supplementary material shows the durability of the solar-cell parameters. All parameters decreased monotonically as the duration of the simulated solar irradiation increased. The decrease in $J_{\mathrm{sc}}$ may be attributed to the carrier scattering due to the formation of the long-lived holes in the spiro-OMeTAD film. The decrease in $V_{\text {oc }}$ may be related to the formation of electric dipole layers due to long-lived charges at the perovskite/spiro-OMeTAD interface. The further investigation into the correlation between the charge states and the device performance using ESR spectroscopy is currently in progress and will be reported in a separate paper.

In summary, we performed the ESR study of the spiroOMeTAD thin films and the perovskite/spiro-OMeTAD layered films, where spiro-OMeTAD were undoped or doped with Li-TFSI, and analyzed the states of long-lived charges in the materials from a microscopic viewpoint. For the spiroOMeTAD thin films, the dramatic increase in the ESR signal due to the hole formation in spiro-OMeTAD was observed by the Li-TFSI doping. The increase in the doping effects was also observed under simulated solar irradiation. For the 
perovskite/spiro-OMeTAD (with Li-TFSI) layered films, the increase in the number of the long-lived holes was observed under the light irradiation, which showed the increase in the ratio of the mobile long-lived holes. The remained holes after turning off the light irradiation were ascribed to the increase in the Li-TFSI doping effects under the light irradiation. Thus, the variation of the charge states in the perovskite solar-cell materials under the light irradiation is demonstrated by the ESR analysis at a molecular level. Such knowledge would be important for understanding of the device operation and the deterioration mechanism of the perovskite solar cells.

See supplementary material for the details of the sample fabrication, the fitting analysis, and the device characteristics of the perovskite solar cells.

This work was supported by JSPS KAKENHI Grant Nos. JP24560004 and JP15K13329, by JST, PRESTO, by SEI Group CSR Foundation, and by JST, ALCA.

${ }^{1}$ M. M. Lee, J. Teuscher, T. Miyasaka, T. N. Murakami, and H. J. Snaith, Science 338, 643 (2012).

${ }^{2}$ D. Liu and T. L. Kelly, Nat. Photonics 8, 133 (2014).

${ }^{3}$ M. Grätzel, Nat. Mater. 13, 838 (2014).

${ }^{4}$ N.-G. Park, Mater. Today 18, 65 (2015).

${ }^{5}$ W. S. Yang, J. H. Noh, N. J. Jeon, Y. C. Kim, S. Ryu, J. Seo, and S. I. Seok, Science 348, 1234 (2015).

${ }^{6}$ M. A. Green, K. Emery, Y. Hishikawa, W. Warta, and E. D. Dunlop, Prog. Photovoltaics: Res. Appl. 24, 3 (2016).

${ }^{7}$ J. H. Noh, S. H. Im, J. H. Heo, T. N. Mandal, and S. I. Seok, Nano Lett. 13, 1764 (2013).

${ }^{8}$ Y. Ogomi, A. Morita, S. Tsukamoto, T. Saitho, N. Fujikawa, Q. Shen, T. Toyoda, K. Yoshino, S. S. Pandey, T. Ma, and S. Hayase, J. Phys. Chem. Lett. 5, 1004 (2014).
${ }^{9}$ Y. Yamada, T. Nakamura, M. Endo, A. Wakamiya, and Y. Kanemitsu, J. Am. Chem. Soc. 136, 11610 (2014).

${ }^{10}$ U. B. Cappel, T. Daeneke, and U. Bach, Nano Lett. 12, 4925 (2012).

${ }^{11}$ A. Abate, T. Leijtens, S. Pathak, J. Teuscher, R. Avolio, M. E. Errico, J. Kirkpatrik, J. M. Ball, P. Docampo, I. McPhersonc, and H. J. Snaith, Phys. Chem. Chem. Phys. 15, 2572 (2013).

${ }^{12}$ W. H. Nguyen, C. D. Bailie, E. L. Unger, and M. D. McGehee, J. Am. Chem. Soc. 136, 10996 (2014).

${ }^{13}$ K. Marumoto, S. Kuroda, T. Takenobu, and Y. Iwasa, Phys. Rev. Lett. 97, 256603 (2006).

${ }^{14}$ K. Marumoto, M. Kato, H. Kondo, S. Kuroda, N. C. Greenham, R. H. Friend, Y. Shimoi, and S. Abe, Phys. Rev. B 79, 245204 (2009).

${ }^{15}$ K. Marumoto, T. Fujimori, M. Ito, and T. Mori, Adv. Energy Mater. 2, 591 (2012).

${ }^{16}$ T. Nagamori and K. Marumoto, Adv. Mater. 25, 2362 (2013).

${ }^{17}$ D. Liu, T. Nagamori, M. Yabusaki, T. Yasuda, L. Han, and K. Marumoto, Appl. Phys. Lett. 104, 243903 (2014).

${ }^{18}$ D. Son, T. Kuwabara, K. Takahashi, and K. Marumoto, Appl. Phys. Lett. 109, 133301 (2016).

${ }^{19}$ Y. Ogomi, A. Morita, S. Tsukamoto, T. Saitho, Q. Shen, T. Toyoda, K. Yoshino, S. S. Pandey, T. Ma, and S. Hayase, J. Phys. Chem. C 118, 16651 (2014).

${ }^{20}$ A. Abate, D. J. Hollman, J. Teuscher, S. Pathak, R. Avolio, G. D'Errico, G. Vitiello, S. Fantacci, and H. J. Snaith, J. Am. Chem. Soc. 135, 13538 (2013).

${ }^{21}$ I.-K. Ding, N. Tétreault, J. Brillet, B. E. Hardin, E. H. Smith, S. J. Rosenthal, F. Sauvage, M. Grätzel, and M. D. McGehee, Adv. Funct. Mater. 19, 2431 (2009).

${ }^{22}$ N. J. Jeon, H. G. Lee, Y. C. Kim, J. Seo, J. H. Noh, J. Lee, and S. I. Seok, J. Am. Chem. Soc. 136, 7837 (2014).

${ }^{23}$ I. A. Shkrob and T. W. Marin, J. Phys. Chem. Lett. 5, 1066 (2014).

${ }^{24}$ H. Nishimura, N. Ishida, A. Shimazaki, A. Wakamiya, A. Saeki, L. T. Scott, and Y. Murata, J. Am. Chem. Soc. 137, 15656 (2015).

${ }^{25}$ N. Ishida, A. Wakamiya, and A. Saeki, ACS Photonics 3, 1678 (2016).

${ }^{26}$ G. Orädd, L. Edman, and A. Ferry, Solid State Ionics 152-153, 131 (2002).

${ }^{27}$ R. Beringer and J. G. Castle, Jr., Phys. Rev. 81, 82 (1951).

${ }^{28}$ M. C. R. Symons, Nature 325, 659 (1987).

${ }^{29}$ R. N. Bagchi, A. M. Bond, F. Scholz, and R. Stösser, J. Am. Chem. Soc. 111, 8270 (1989). 